\title{
THE SINO-GERMAN $\lambda 6$ CM POLARIZATION SURVEY OF THE GALACTIC PLANE: A SUMMARY
}

\author{
J. L. HAN*, ${ }^{*}$, W. REICH ${ }^{\dagger, \S}$, X. H. SUN ${ }^{*} \dagger$, X. Y. GAO*, L. XIAO*, \\ W. B. SHI* ${ }^{*}$ P. REICH ${ }^{\dagger}$ and R. WIELEBINSKI ${ }^{\dagger}$ \\ *National Astronomical Observatories, Chinese Academy of Sciences, \\ Jia-20 Datun Road, Chaoyang District, Beijing 100012, China \\ ${ }^{\dagger}$ Max-Planck-Institut für Radioastronomie, \\ Auf dem Hügel 69, 53121 Bonn, Germany \\ †hl@nao.cas.cn \\ §wreich@mpifr-bonn.mpg.de
}

\begin{abstract}
We have finished the $\lambda 6 \mathrm{~cm}$ polarization survey of the Galactic plane using the Urumqi $25 \mathrm{~m}$ radio telescope. It covers $10^{\circ} \leq l \leq 230^{\circ}$ in Galactic longitude and $|b| \leq 5^{\circ}$ in Galactic latitude. The new polarization maps not only reveal new properties of the diffuse magnetized interstellar medium, but also are very useful for studying individual objects such as Hil regions, which may act as Faraday screens with strong regular magnetic fields inside, and supernova remnants for their polarization properties and spectra. The high sensitivity of the survey enables us to discover two new SNRs G178.2-4.2 and G25.3-2.1 and a number of HiI regions.
\end{abstract}

Keywords: Interstellar medium (ISM) and nebulae in the Milky Way; supernova remnants; HiI regions.

PACS numbers: 98.38.-j, 98.38.Mz, 98.58.Hf

\section{Introduction}

A polarization survey of diffuse Galactic emission provides a direct image of the transverse magnetic field, if Faraday rotation is negligible. The Sino-German $\lambda 6 \mathrm{~cm}$ polarization survey of the Galactic plane was set as the main task for the partner group of the Max-Planck-Institut für Radioastronomie (MPIfR) at the National Astronomical Observatories of Chinese Academy of Sciences (NAOC), which was commissioned in 2001.

Previously the Effelsberg $100 \mathrm{~m}$ telescope was used to map the Galactic polarized emission up to medium latitudes ${ }^{34,21}$ at $1.4 \mathrm{GHz}$ with an angular resolution of 9.4'. Part of the data were used to compensate the missing large-scale structures in the Canadian Galactic Plane Survey (CGPS) ${ }^{19}$ observed with the DRAO synthesis telescope at $1^{\prime}$ angular resolution. At $1.4 \mathrm{GHz}$, mainly polarized emission from the local interstellar medium is observed. ${ }^{6,34}$ The polarization survey by the Westerbork Synthesis Radio Telescope at $350 \mathrm{MHz}^{13,14}$ penetrates through an even shorter distance into the magneto-ionic medium. Earlier 2.7 GHz Galactic plane polarization 
surveys were made with the Effelsberg $100 \mathrm{~m}$ telescope ${ }^{17,2}$ at $4.3^{\prime}$ resolution tracing more distant emission. The Galactic plane in the southern sky was surveyed by the Parkes telescope at $2.4 \mathrm{GHz},{ }^{1}$ while sections of the Galactic plane were observed with the Australian Telescope Compact Array at $1.4 \mathrm{GHz}$ with a high angular resolution. ${ }^{6,12}$ The observing frequencies of previous polarization surveys are all lower than $3 \mathrm{GHz}$, which implies a small distance to the polarization horizon. In other words, the results suffer significant depolarization for distant emission. To penetrate even deeper into the magneto-ionic medium, observations at higher frequencies are required.

Using the Urumqi $25 \mathrm{~m}$ radio telescope, the Sino-German $\lambda 6 \mathrm{~cm}$ polarization survey covers the Galactic plane from $10^{\circ} \leq l \leq 230^{\circ}$ and $|b| \leq 5^{\circ}$ with an angular resolution of $9.5^{\prime}$, which enables us to see through the complex magneto-ionized structures even for a $R M$ of several hundred $\mathrm{rad} \mathrm{m}^{-2}$. In the inner Galaxy of $10^{\circ} \leq l \leq 60^{\circ}$, the radio emission at this frequency comes from diffuse emission and individual objects of a few spiral arms. ${ }^{16}$ Towards the direction of $l \sim 80^{\circ}$, the line of sight is tangential to the local arm, and the large-scale magnetic fields run parallel to the line of sight. There are many objects and structures of different distances in this projected area, forming a radio complex. In the Galactic anti-center region $\left(120^{\circ} \leq l \leq 230^{\circ}\right)$, the emission comes from the Perseus arm and maybe also a weaker outer arm, where the large-scale magnetic fields run almost perpendicular to the line of sight.

\section{Instrumental Setup and Commissioning}

The Sino-German $\lambda 6 \mathrm{~cm}$ polarization survey of the Galactic plane has been conducted using the Urumqi $25 \mathrm{~m}$ telescope of Xinjiang (former Urumqi) Astronomical Observatory, Chinese Academy of Sciences. The telescope is located about $70 \mathrm{~km}$ south of Urumqi city at an altitude of $2029 \mathrm{~m}$ above sea level with the geographic longitude $87^{\circ} \mathrm{E}$ and latitude $43^{\circ} \mathrm{N}$.

The dual-channel $\lambda 6 \mathrm{~cm}$ receiver was designed by Mr. Otmar Lochner and constructed at MPIfR in Germany, based on a receiver used at the Effelsberg $100 \mathrm{~m}$ telescope. The new receiver has a higher stability and a lower $1 /$ f-noise. In 2004, the receiver was installed at the secondary focus of the Urumqi $25 \mathrm{~m}$ telescope. The $\lambda 6$ $\mathrm{cm}$ system includes a corrugated feed horn and an orthogonal transducer to convert the signal into left-hand (L) and right-hand (R) polarization channels. The signal of the two channels is amplified by cooled HEMT pre-amplifiers working below $15 \mathrm{~K}$ in a dewar and then mixed with the local oscillator signal. Two splitters follow, which split one set of $\mathrm{L}$ and $\mathrm{R}$ channels into two sets, one used to directly detect the power of $L=\mathrm{LL}^{*}$ and $R=\mathrm{RR}^{*}$ for Stokes $I$, and the other one is feed into the IF polarimeter for producing $\mathrm{LR}^{*}$ and $\mathrm{RL}^{*}$ for the Stokes $U$ and $Q$. The voltage-frequency converters in the focus cabin are used for converting the detected voltage signals to frequencies, which enables the long-distance transportation of the signals to a digital backend in the observatories control room. A MPIfR pocket backend counts 
Table 1. Survey parameters of the Urumqi $\lambda 6 \mathrm{~cm}$ system.

\begin{tabular}{ll}
\hline Frequency $[\mathrm{MHz}]$ & $4800 / 4963$ \\
Bandwidth $[\mathrm{MHz}]$ & $600 / 295$ \\
$\mathrm{~T}_{\text {sys }}[\mathrm{K}] \mathrm{T}_{a}$ & 22 \\
$\mathrm{HPBW}\left[{ }^{\prime}\right]$ & 9.5 \\
Scan direction & $l$ and $b$ \\
& $\mathrm{RA}$ and DEC \\
$\mathrm{T}_{B} / \mathrm{S}$ (Gain) & $0.164 \mathrm{~K} / \mathrm{Jy}$ \\
First side lobe & $<2 \%$ \\
Instrumental polarization & $<2 \%$ \\
$\sigma_{I}[\mathrm{mK}] \mathrm{T}_{b}$ & 1.0 \\
$\sigma_{P I}[\mathrm{mK}] \mathrm{T}_{b}$ & 0.4 \\
\hline
\end{tabular}

the frequency-coded signals from the four polarization channels $\left(\mathrm{RR}^{*}, \mathrm{LL}^{*}, \mathrm{RL}^{*}\right.$, $\mathrm{LR}^{*}$ ) at a sampling rate of $32 \mathrm{msec}$. Every $32 \mathrm{msec}$ the frontend setting changes, so that either a calibration signal of $1.7 \mathrm{~K} \mathrm{~T}_{\mathrm{a}}$ is added to the antenna signal and/or the signal phase is shifted by $180^{\circ}$. For two subsequent phases within a cycle, the calibration signal is switched on, so that any gain changes of the system can be monitored. The phase of the output signals is switched by $180^{\circ}$ alternatively for every phase, so that the quadratic terms of the polarimeter are canceled. In one whole cycle of $128 \mathrm{~ms}$, four combinations of different settings of calibration and phase-switching are realized.

The raw data are transfered to a Linux PC via the TCP/IP protocol and stored on disk for further processing in the MBFITS format developed for the Effelsberg $100 \mathrm{~m}$ telescope. By using the TOOLBOX-software package, the raw data of the four backend channels $\left(\mathrm{RR}^{*}, \mathrm{LL}^{*}, \mathrm{RL}^{*}, \mathrm{LR}^{*}\right)$ are converted from a time series into a tabulated format with a user specified spatial separation on the sky using a sincinterpolation function. The calibration signals of each subscan (row or column) of a map are extracted and fitted to account for any gain drifting and also to control the phase stability of the IF polarimeter. The tabulated maps are then transformed into NOD2 maps $^{11}$ with the polarization $U$ and Q-channels corrected for parallactic angles. The Linux PC is also used to command the telescope control computer to move the telescope in a number of different astronomical coordinate systems (RA/DEC or $l / b)$ and in the azimuth/elevation coordinate system of the telescope.

Test observations were made in August 2004, and the relevant system and antenna parameters were determined (see Table 1). By periodically injecting a known signal, we got the system temperature measured as $22 \mathrm{~K} \mathrm{~T}_{a}$ when the $\lambda 6 \mathrm{~cm}$ receiver is pointing to the zenith of a clear sky. The strong radio source $3 \mathrm{C} 286$ is used to determine other parameters, such as the beam size, effective collecting area, main beam efficiency and gain. Indian satellites which are known to emit strong in the $\lambda 6 \mathrm{~cm}$ band were observed to estimate the instrumental effects, e.g. the side lobes. The unpolarized radio source 3C 295 was used to calculate the leakage of the total intensity into the polarization channels, namely the instrumental polarization. From these tests, we found the $\lambda 6 \mathrm{~cm}$ beam is rather symmetric and the first side 
lobes, are less than $2 \%$ of the main beam. Cross-scans were made in two orthogonal directions to the source $3 \mathrm{C} 286$, and showed that the pointing accuracy of the system is stable and the offset is negligible in comparison with the beam size.

During the test observations, we identified internal and external interferences. TThe observatory's pulsar filter-bank backend introduced the major internal interference, and was switched off during the $\lambda 6 \mathrm{~cm}$ observations. The external interferences came from the ground radiation and the Indian satellites. The Nanshan mountains around the site induce significant ground radiation picked up by the sidelobes of the telescope, which produce a non-flat baseline for scans of low elevations. ${ }^{35}$ For the required survey quality, observations must be done at elevations larger than $20^{\circ}$, so that the baseline from the ground radiation can be well subtracted by a first-order or second-order polynomial fit. There are four locations of Geostationary Indian satellites, which emit strong signals in the $\lambda 6 \mathrm{~cm}$ band, were observed at the azimuths and elevations of $\left(222.61^{\circ}, 30.34^{\circ}\right),\left(198.53^{\circ}, 38.19^{\circ}\right)$, $\left(186.06^{\circ}, 39.7^{\circ}\right)$, and $\left(170.85^{\circ}, 39.4^{\circ}\right)$, which emit strong signals up to $4810 \mathrm{MHz}$ with a flux density of $>60000 \mathrm{Jy}$. Our $\lambda 6 \mathrm{~cm}$ system suffers from their signals even tens of degrees away via telescope side lobes. In November 2005, the receiver was equipped with a switched bandpass filter. When it is switched on, the lower band is cut off, and the central observing frequency is shifted from $4800 \mathrm{MHz}$ to $4963 \mathrm{MHz}$, and the bandwidth is cut from $600 \mathrm{MHz}$ to $295 \mathrm{MHz}$. We have to use this narrow band to observe the southern parts of the Galactic plane.

\section{Observations and Data Reduction}

The $\lambda 6 \mathrm{~cm}$ system is very sensitive recording, for instance, any vibration of the thin RF-transparent membrane of of the focus-cabin by wind or small temperature variations in the focus cabin, which was fixed. However, effects by a bird or clouds passing the telescope beam and, in particular, emission from the sun picked up by the telescope side lobes could not be fixed. Therefore, the survey observations were always carried out in clear nights.

The $\lambda 6 \mathrm{~cm}$ survey observations started in August 2004, and finished in April 2009. A very large section of the Galactic plane of 2200 square degrees for $10^{\circ} \leq l \leq$ $230^{\circ}$ and $|b| \leqslant 5^{\circ}$ has been scanned in two orthogonal directions: along the Galactic longitude for the $l$ map and along the Galactic latitude for the $b$ map. Considering the stability of the system and the variable ground radiation, scans must be done for a finite size in a given range of the sky. For a $l$ map, the typical map size of $l \times b$ is $8.6^{\circ} \times 2.6^{\circ}$; for a $b$ map it is $2.2^{\circ} \times 10.0^{\circ}$. There is always a small overlap of the different patches. Before and after observations for each map, calibrators are observed. 3C 286 served as our main calibrator. It has an integrated flux density of $S_{6 \mathrm{~cm}}=7.5 \mathrm{Jy}$ at $\lambda 6 \mathrm{~cm}, 11.3 \%$ polarization fraction, and a polarization angle of $33^{\circ}$. 3C 48 and $3 \mathrm{C} 138$ were used as secondary calibrators. The unpolarized radio source 3C 295 was also frequently observed for the cleaning of the instrumental polarization. 
As mentioned above, the original survey data were stored in a Linux PC. We used the "toolbox" and "mapax" software originally developed at the MPIfR for the data reduction of Effelsberg continuum and polarization observations. There are several steps in data processing. The maps were first edited to remove spiky interference and obvious very bad scans due to interference. Then we corrected baseline curvatures by polynomial fitting and suppressed scanning effects by applying an "unsharp masking method". ${ }^{24}$ All individual maps were therefore on a relative baselevel, where the two ends of each subscan were set to zero. The instrumental polarization, mainly the leakage of total power emission into the polarization channels, was determined by observations of the unpolarized calibrators 3C 295 and 3C 147, and corrected using the "REBEAM" method. ${ }^{25}$ Then the intensity calibrations was made to each map. Finally, we weaved the processed $l$ maps and $b$ maps together by using the "PLAIT" method, ${ }^{3}$ which further reduced remaining scanning effects, to form the Stokes $I, U$, and $Q$ survey maps. The survey strategy together with the results from a test region of $122^{\circ} \leq l \leq 129^{\circ}$ was published in the first survey paper. ${ }^{26}$

\section{Results}

For every sky area, in general, we performed observations for more than one $l$ map and $b$ map. Combining all observations together, we obtained the $I, U$, and $Q$ maps for the whole survey region. The r.m.s.-noise in the $I$ map is about $1.0 \mathrm{mK} \mathrm{T}_{b}$, and in the $U$ and $Q$ maps it is about $0.5 \mathrm{mK} \mathrm{T}_{b}$. All survey data have been released at the end of $2010^{\mathrm{a}}$. See Fig. 1 for a section of survey maps. It is by far the highest frequency in a ground-based polarization Galactic plane survey.

\subsection{Survey maps}

The total intensity maps show much stronger diffuse emission towards the inner Galaxy $\left(l<60^{\circ}\right)$ originating from spiral arms. ${ }^{29}$ For the mixed emission in the inner region of the Galactic plane, by using the multi-frequency total power data together with our $\lambda 6 \mathrm{~cm}$ map, we separated the thermal bremsstrahlung emission component and the non-thermal synchrotron emission component, ${ }^{29}$ we found that the thermal emission is about $60 \%$ of the detected $\lambda 6 \mathrm{~cm}$ emission. In the outer Galaxy, ${ }^{37}$ the most outstanding region is the Cygnus complex located at about $77^{\circ}<l<87^{\circ}$ and $-2^{\circ}<b<4^{\circ}$, where indistinguishable radio emission from HiI regions and other objects accumulates in the tangential direction of the local arm. ${ }^{16}$ In the anti-centre region of $l>120^{\circ}$, most prominent are large HiI regions and supernova remnants (SNRs) in the Perseus arm. ${ }^{8}$

When eliminating the ground radiation with polynomial fits as stated above, polarized emission with spatial scale of the scan length of each small survey area is filtered out. Absolute baselevels for $U$ and $Q$ are important to calculate correct polarization angles and intensities. Missing large-scale components leads to a

ahttp://zmtt.bao.ac.cn/6cm/ 
misinterpretation of polarization data. For the $\lambda 6 \mathrm{~cm}$ survey, the ongoing $\mathrm{C}$-Band All-Sky Survey (CBASS) might provide the missing large-scale emission. However, CBASS data are not yet available. The best we can do at present is to restore the large-scale $U$ and $Q$ components at $\lambda 6 \mathrm{~cm}$ using the WMAP K-band $(22.8 \mathrm{GHz})$ polarization data. ${ }^{20,15}$ The spectral index of the polarization intensity of the synchrotron emission in the plane varies from $\beta=-3.1$ to $\beta=-2.7$. The $U$ and $Q$ maps of high-latitude regions at $22.8 \mathrm{GHz}$ were scaled to $\lambda 6 \mathrm{~cm}(4.8 \mathrm{GHz})$ according to the spectral index $\beta$ to restore the baselevel of $\lambda 6 \mathrm{~cm} U$ and $Q$ maps, from which the $P I$ and $P A$ maps are obtained.

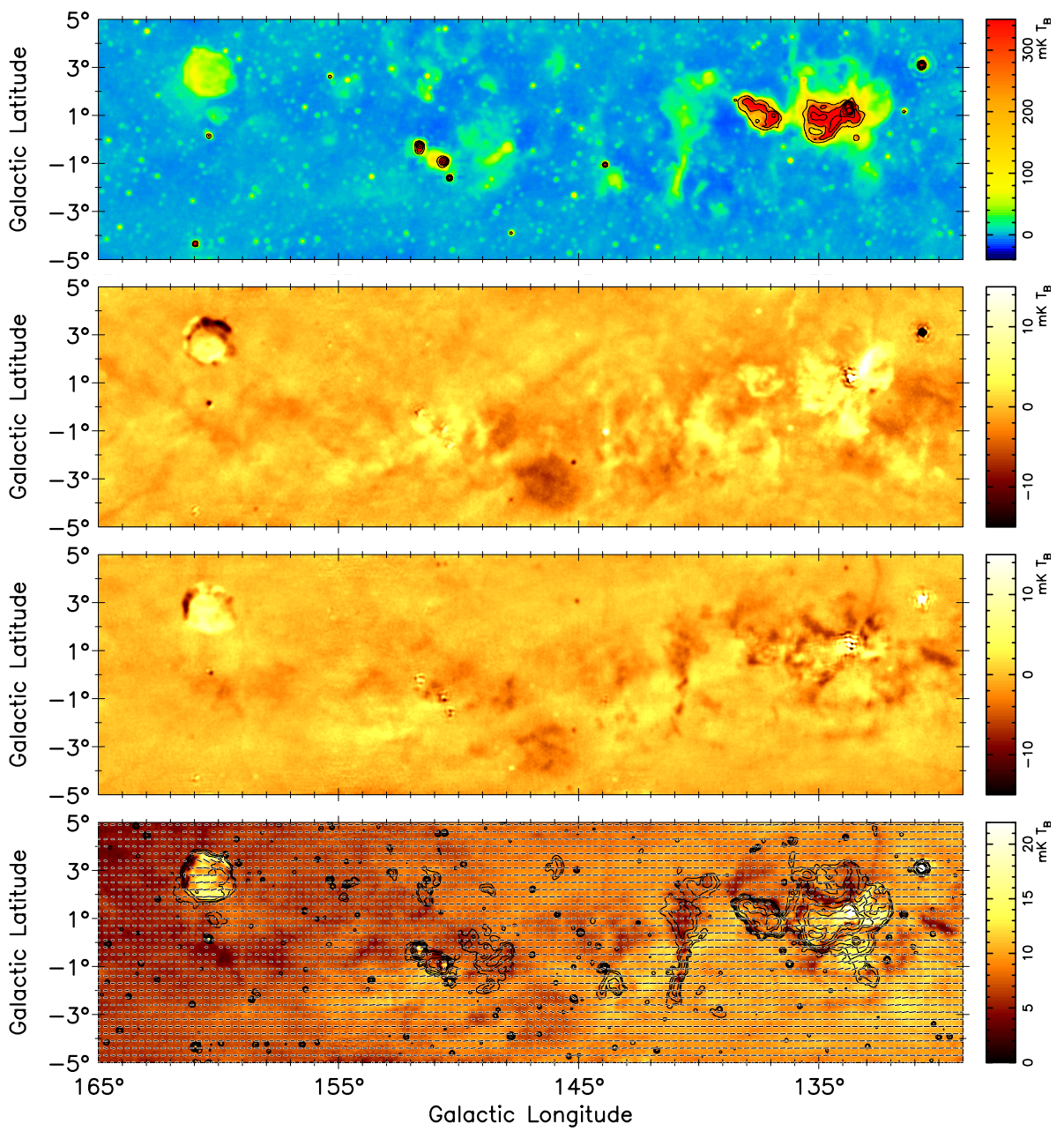

Fig. 1. A section of survey map. From top to bottom are the observed total intensity $I$ map, and Stokes $U$ and $Q$ maps. The $P I$ map includes the large scale emission with $P A+90^{\circ}$ vectors and contours of total power overlaid. 
From the polarization map of the survey area, we found many polarization patches and depolarization structures of different scales. ${ }^{8,29,37}$ The prominent objects are again large HII regions because of their depolarization and SNRs because of their polarized emission. There are also many polarized spurs and "depolarized canals". A few large polarized patches with an angular size of a few degrees are probably discrete features within $1 \mathrm{kpc}$. We have studied the fluctuation properties in the $U, Q$, and $P I$ maps in the survey region of $10^{\circ} \leq l \leq 230^{\circ}$ by a structure function analysis. ${ }^{37}$ Although the indices of the structure functions are always close to about 0.5 , the fluctuation power is much lower for the anti-center region. More enhanced polarization structures on small scales give much more fluctuation power towards the inner Galaxy. ${ }^{37}$ The $\lambda 6 \mathrm{~cm}$ polarization horizon in the inner Galaxy is about $4 \mathrm{kpc},{ }^{29}$ which means that the observed polarized radiation comes from the Sagittarius and Scutum arm.

\subsection{HII regions and Faraday screens}

Large HII regions are prominent radio sources in the $\lambda 6 \mathrm{~cm}$ total power map. In our polarization map, they often appear due to their depolarization and Faraday rotation.

Along the periphery of many large Hiı regions (a few degrees in size), very clear depolarization has been found in our restored polarization map. ${ }^{37}$ This indicates either strong magnetic fields or large electron density towards the boundaries of HII regions. The regular magnetic fields in HII regions have been studied recently by using the rotation measure data. ${ }^{10} \mathrm{~A}$ detailed modelling is in progress.

Polarization angles in some regions of degrees in size significantly deviate from the surroundings, ${ }^{26,8,37,29}$ which are caused by foreground Faraday screens. These screens have a very small electron density and therefore cannot be detected in total intensity. Our modeling shows that the line of sight component of the magnetic fields in the screens is up to tens of $\mu \mathrm{G}$, much stronger than the average interstellar magnetic fields. The nature of Faraday screens remains unclear.

\subsection{Measurements of known supernova remnants}

Because of the intermediate beam size of the $\lambda 6 \mathrm{~cm}$ observation system, we have advantage to map large SNRs of several degrees in size, which is a very ambitious task for large radio telescopes with very small beams.

The first $\lambda 6 \mathrm{~cm}$ test observation ${ }^{28}$ was carried out to the large SNR Cygnus loop (G74.0-8.5), with the apparent size of $3^{\circ} \times 4^{\circ}$. We obtained a total intensity image, from which we derived the integrated flux density at $\lambda 6 \mathrm{~cm}$ and the radio spectrum from $408 \mathrm{MHz}$ to $4.8 \mathrm{GHz}$ when combined with the flux densities from the literature. The spectrum does not show any steepening. Our new $\lambda 6 \mathrm{~cm}$ polarization image indicates a distinct magnetic field configuration for the northern and southern part of the SNR, which may support the idea for the Cygnus Loop resulting from two smaller SNRs. ${ }^{33}$ 
In the survey test region ${ }^{26}$ of $122^{\circ}<l<129^{\circ}$, we studied the SNRs G126.2+1.6 and G127.1+0.5. For the former we excluded the suggested spectral break ${ }^{32}$ at about $1 \mathrm{GHz}$. We have also studied 17 supernova remnants in the survey region which are larger than $1^{\circ}$ in the fifth survey paper ${ }^{7}$ and 50 smaller ones in the seventh survey paper. ${ }^{27}$ Using the Urumqi data, we determined the flux densities at $\lambda 6 \mathrm{~cm}$. Together with the flux density data at other frequencies from literature, we derived the radio spectra of these SNRs. A number of the previously reported flux densities and spectral indices were corrected/improved by our new measurements.

Some large SNRs are outside the survey region. We have also observed them and studied individually. Besides the Cygnus Loop, ${ }^{28}$ the faintest SNR in the Galaxy, G156.2+5.7, ${ }^{39}$ was imaged in total intensity and polarization by Urumqi $\lambda 6 \mathrm{~cm}$ and Effelsberg $\lambda 11 \mathrm{~cm}$ observations. A highly polarized central patch is detected, and the toroidal magnetic field configuration is identified for the SNR. For the large SNR in the anti-center region of the Galactic plane, S147, the high quality Urumqi $\lambda 6 \mathrm{~cm}$ data confirm the spectral break $^{5}$ of the $\mathrm{SNR}$ at about $1.5 \mathrm{GHz}{ }^{36}$ Two populations of electrons with different energy spectral indices are responsible for the diffuse emission and the filamentary emission component. Using the Urumqi $\lambda 6 \mathrm{~cm}$ flux density data, together with Effelsberg $\lambda 11 \mathrm{~cm}$ and $\lambda 21 \mathrm{~cm}$ data (see http: //www.mpifr.de /survey.html), we clarify that SNR HB3 has a straight spectrum $^{23}$ with a spectral index of $\alpha=-0.61 \pm 0.06$ between $1.4 \mathrm{GHz}$ and $4.8 \mathrm{GHz}$, which is consistent with that at frequencies below $1 \mathrm{GHz}^{18}$ and rejected the suggested spectral flattening above 1 GHz. ${ }^{31}$ For another faint SNR G65.2+5.7, we obtained the integrated radio spectral index from $83 \mathrm{MHz}$ to $4.8 \mathrm{GHz}^{38}$ and estimated the magnetic field strength in the southern filamentary shell using new $\lambda 6$ $\mathrm{cm}$ and Effelsberg $\lambda 11 \mathrm{~cm}$ polarization data. Using the polarization maps of the Urumqi $\lambda 6 \mathrm{~cm}$ and Effelsberg $\lambda 11 \mathrm{~cm}$ observations, together with data in literature, the spectral index and its distribution as well as the magnetic fields of the shell and central branch of SNR CTA 1 were studied. ${ }^{30}$ A possible foreground Faraday screen partly covering CTA 1 was identified from the rotation measure distribution.

In summary, of all $(\sim 80)$ known SNRs we investigated with the Urumqi data, for $10 \%$ of them polarization was newly detected and $35 \%$ of them the integrated flux densities were measured for the first time at $\lambda 6 \mathrm{~cm}$. The Urumqi data are at the highest frequency for more than $50 \%$ of the SNRs, which play an important role in determining their spectral indices. The survey gives the first (complete) polarization images for about $30 \%$ of the SNRs, which is important to understand the magnetic field structure of the SNRs.

Using the high-quality data of the Urumqi $\lambda 6 \mathrm{~cm}$ and Effelsberg $\lambda 11 \mathrm{~cm}$ and $\lambda 21 \mathrm{~cm}$ data, we have disproved three objects, G192.8-1.1, ${ }^{7} \mathrm{G} 166.2+2.5$ (OA184), ${ }^{4}$ and G16.8-1.1, ${ }^{27}$ as being SNRs. Their emission turns out to be thermal without any polarization, and hence they are very probably Hı regions. 


\subsection{Discoveries of new SNRs and HII regions}

Sensitive surveys always lead to new discoveries, so does the Urumqi $\lambda 6 \mathrm{~cm}$ survey. With the high sensitivity of the $\lambda 6 \mathrm{~cm}$ system, we were able to discover two new shell-type SNRs: G178.2-4.2 and G25.1-2.3. ${ }^{9}$ G178.2-4.2 has a size of $72^{\prime} \times 62^{\prime}$ with strongly polarized emission along its northern shell. The spectral index of G178.2-4.2 is $\alpha=-0.48 \pm 0.13$. Its surface brightness is $\Sigma_{1} \mathrm{GHz}=$ $7.2 \times 10^{-23} \mathrm{Wm}^{-2} \mathrm{~Hz}^{-1} \mathrm{sr}^{-1}$, which ranks it the second faintest known Galactic SNR. G25.1-2.3 is revealed by its strong southern shell, which has a size of $80^{\prime} \times 30^{\prime}$. It has a non-thermal radio spectrum with a spectral index of $\alpha=-0.49 \pm 0.13$.

We have also identified a number of HiI regions. The first is G124.9+0.1 (30') in the test survey region, ${ }^{26}$ which acts as a Faraday screen. Its regular magnetic field parallel to the line-of-sight can thus be estimated to be about $3.9 \mu \mathrm{G}$. Other new Hit regions are G12.83.6 $\left(100^{\prime} \times 24^{\prime}\right),{ }^{29} \mathrm{G} 56.70 .6\left(65^{\prime} \times 27^{\prime}\right),{ }^{29} \mathrm{G} 98.3-1.6$ $\left(90^{\prime} \times 66^{\prime}\right),{ }^{37} \mathrm{G} 119.6+0.4\left(44^{\prime} \times 44^{\prime}\right),{ }^{37} \mathrm{G} 148.8+2.3\left(63^{\prime} \times 40^{\prime}\right),{ }^{22} \mathrm{G} 149.5+0.0\left(30^{\prime}\right)^{22}$ and G169.9+2.0 $\left(27^{\prime} \times 20^{\prime}\right) .{ }^{22}$ A more detailed study of HiI regions from the survey is being prepared (Gao et al. in prep.)

\section{Conclusions}

Our new polarization survey at $\lambda 6 \mathrm{~cm}$ can delineate the Galactic magnetic fields in both large and small scales, in a larger and deeper area than that observed at low radio frequencies. With its high quality data, we studied the radio properties of many known SNRs covered by the survey. We discovered two new SNRs with very faint surface brightness. We revealed several prominent Faraday screens with high RM values, HiI regions, and many interesting polarization/depolarization structures, e.g. depolarization around the edge of ionized HiI regions, canals, and polarization patches.

\section{Acknowledgments}

We thank Drs. Hui Shi and Chen Wang for conducting some observations. The Sino-German $\lambda 6 \mathrm{~cm}$ polarization survey was carried out with a receiver system constructed by Mr. Otmar Lochner at MPIfR with financial support by the MPG and the NAOC. We thank Dr. Peter Müller for the installation and adaptation of Effelsberg data reduction software at the Urumqi observatory, and Mr. Maozheng Chen and Jun Ma for operation support and maintenance, and also Prof. Ernst Fürst for a lot of help and involvement in the project. The survey team was supported by the National Natural Science foundation of China (10773016, 10821061, and 10833003).

\section{References}

1. A. R. Duncan, R. F. Haynes, K. L.Jones, \& R. T. Stewart, MNRAS 291, 279 (1997).

2. A. R. Duncan, P. Reich, W. Reich, \& E. Fürst, A\&SA 350, 447 (1999).

3. D. T. Emerson, \& R. Gräve, A\&A 190, 353 (1988). 
4. T. Foster, R. Kothes, X. H. Sun, W. Reich, J. L. Han, A $\& A$ 454, 517 (2006)

5. E. Fürst, \& W. Reich, A\&A 163, 185 (1986).

6. B. M. Gaensler, J. M. Dickey, N. M. McClure-Griffiths, A. J. Green, M. H. Wieringa, R. F. Haynes, ApJ 549, 959 (2001).

7. X. Y. Gao, J. L. Han, W. Reich, P. Reich, X. H. Sun, L. Xiao, A $\& A$ 529, A159 (2011a).

8. X. Y. Gao, W. Reich, J. L. Han, X. H. Sun, R. Wielebinski, W. B. Shi, L. Xiao, P. Reich, E. Fürst, M. Z. Chen, J. Ma, A\&SA 515, A64 (2010).

9. X. Y. Gao, X. H. Sun, J. L. Han, W. Reich, P. Reich, R. Wielebinski, A $\& A$ 532, A144 (2011b).

10. L. Harvey-Smith, G. J. Madsen, B.M. Gaensler, ApJ 736, 83 (2011).

11. C. G. T. Haslam, A $\mathscr{E} A S$ 15, 333 (1974).

12. M. Haverkorn, B. M. Gaensler, N. M. McClure-Griffiths, J. M. Dickey, \& A. J. Green, ApJS 167, 230 (2006).

13. M. Haverkorn, P. Katgert, \& A. G. de Bruyn, A\&A 403, 1031 (2003a).

14. M. Haverkorn, P. Katgert, \& A. G. de Bruyn, A\&SA 404, 233 (2003b).

15. G. Hinshaw, J. L. Weiland, R. S. Hill, et al., ApJS 180, 225 (2009).

16. L. G. Hou, J. L. Han, \& W. B. Shi, A\&A 499, 473 (2009).

17. N. Junkes, E. Fürst, \& W. Reich, $A \& A S$ 69, 451 (1987).

18. R. Kothes, K. Fedotov, T. J. Foster, \& B. Uyanıker, A $\mathscr{\mho} A$ 457, 1081 (2006).

19. T. L. Landecker, W. Reich, R. I. Reid, et al., A\&A 520, A80 (2010).

20. L. Page, G. Hinshaw, E. Komatsu, M. R. Nolta, et al., ApJS 170, 335 (2007).

21. W. Reich, E. Fürst, P. Reich, B. Uyanıker, R. Wielebinski, M. Wolleben, in: "The magnetized interstellar medium", eds. B. Uyanıker, W. Reich, R. Wielebinski, (Copernikus GmbH, Katlenburg-Lindau, 2004), p. 51

22. W. B. Shi, X. Sun, J. Han, X. Gao, \& L. Xiao, Chinese Journal of Astronomy and Astrophysics 8, 575 (2008a).

23. W. B. Shi, J. L. Han, X. Y. Gao, X. H. Sun, L. Xiao, P. Reich, W. Reich, A\&A 487, $601(2008 b)$.

24. Y. Sofue, \& W. Reich, A\&AS 38, 251 (1979).

25. Y. Sofue, W. Reich, M. Inoue, \& J.H. Seiradakis, PASJ 39, 95 (1987).

26. X. H. Sun, J. L. Han, W. Reich, P. Reich, W. B. Shi, R. Wielebinski, E. Fürst, $A \mathscr{E} A$ 463, 993 (2007).

27. X. H. Sun, P. Reich, W. Reich, L. Xiao, X. Y. Gao, J. L. Han, A\&A A 536, A83 (2011a).

28. X. H. Sun, W. Reich, J. L. Han, P. Reich, \& R. Wielebinski, A\&A 447, 937 (2006).

29. X. H. Sun, W. Reich, J. L. Han, P. Reich, R. Wielebinski, C. Wang, P. Müller, $A \& A$ 527, A74 (2011b).

30. X. H. Sun, W. Reich, C. Wang, J. L. Han, \& P. Reich, AEGA 535, A64 (2011c).

31. W. W. Tian, \& D. A. Leahy, A\&A 436, 187 (2005).

32. W. W. Tian, \& D. A. Leahy, $A \mathscr{E} A$ 447, 205 (2006).

33. B. Uyaníker, R. Kothes, \& C. M. Brunt, ApJ 565, 1022 (2002).

34. B. Uyanıker, E. Fürst, W. Reich, P. Reich, \& R. Wielebinski, A\&AS 138, 31 (1999).

35. C. Wang, J. L. Han, X. H. Sun, W. B. Shi, M. Z. Chen, J. Ma, Astronomical Research and Technology, 4, 181 (2007).

36. L. Xiao, E. Fürst, W. Reich, \& J. L. Han, A\&A 482, 783 (2008).

37. L. Xiao, J. L. Han, W. Reich, X. H. Sun, R. Wielebinski, P. Reich, H. Shi, O. Lochner, AछA 529, A15 (2011).

38. L. Xiao, W. Reich, E. Fürst, \& J. L. Han, A\&A 503, 827 (2009).

39. J. W. Xu, J. L. Han, X. H. Sun, W. Reich, L. Xiao, P. Reich, and R. Wielebinski $A \mathscr{E} A$ 470, 969 (2007). 